\title{
RICERCA
}

\section{L'UTILIZZO DEI MEDIA DIGITALI IN UN CAMPIONE DI BAMBINI E ADOLESCENTI ATTRAVERSO IL QUESTIONARIO MEDIA ACTIVITY FORM}

\author{
Eleonora Rosi, Paola Colombo, Massimo Molteni, Nicole Viganò, Alessandra Frigerio \\ IRCCS Eugenio Medea, Bosisio Parini, Lecco
}

Indirizzo per corrispondenza: alessandra.frigerio@lanostrafamiglia.it

\section{THE USE OF DIGITAL MEDIA THROUGH THE MEDIA ACTIVITY FORM IN A SAMPLE OF ITALIAN CHILDREN AND ADOLESCENTS}

\section{Key words}

Digital media, Social network, Children, Adolescents

\begin{abstract}
Background - Several studies have investigated the use of digital media in developmental age, mainly focusing on Internet or video games addiction in adolescence. However, few studies have investigated the time spent by children and young people on different types of digital media and their impact on adaptive behaviour.

Objectives - On the base of parent and child reports the current study aimed to investigate the use of different digital media in Italian children and adolescents, aged six to eighteen, and their impact on adaptive behaviour as well, and to assess its association with socio-demographic factors.

Material and methods - 901 parents and 574 children were recruited in ten schools in Northern Italy. The parents filled in a socio-demographic form and the "Media Activity Form" (MAF-G), while adolescents filled in a youth version of the "Media Activity Form" (MAF-R).

Results - Overall, the activities carried out by children (6-11 years old) are mainly focused on viewing films/TV series and using video games, while those of adolescents are mainly related to listening to music and messaging. Parents estimated a lower amount of time spent on digital media by their children compared to that reported by their children. Finally, significant differences emerged in relation to male gender, preadolescence, low socioeconomic level, low maternal education and divorced/separated parents, showing that participants belonging to such categories spent longer time on different digital media.
\end{abstract}

Conclusions - The results contributed to improve the knowledge on the use of digital media in children and adolescents, with useful clinical implications for interventions.

\section{RIASSUNTO}

Razionale - Esistono diversi studi sull'utilizzo dei media digitali in età evolutiva, principalmente focalizzati sulla dipendenza da Internet o da videogiochi nella fascia di età adolescenziale. Sono pochi, invece, gli studi che hanno analizzato la quantità di tempo che bambini e ragazzi trascorrono su diverse tipologie di media digitali e il loro impatto sul funzionamento adattivo.

Obiettivi - L'obiettivo principale di questo studio è analizzare, secondo la percezione del genitore e quella del ragazzo, l'utilizzo di diversi media digitali nei bambini e adolescenti italiani, di età compresa tra i sei e i diciotto anni, e il loro impatto sul funzionamento adattivo, valutando anche $\mathrm{i}$ fattori di rischio socio-demografici a esso associati.

Materiali e metodi - Il campione è costituito da 901 genitori e 574 ragazzi reclutati attraverso 10 scuole di ogni grado scolastico (primaria, secondaria di primo e secondo grado) del Nord Italia. Ai genitori è stato richiesto di compilare una scheda sui dati socio-demografici e il questionario Media Activity Form (MAF); anche i ragazzi, a partire dagli 11 anni di età, compilavano la versione per giovani del questionario MAF.

Risultati - In generale, le attività svolte dai bambini (6-11 anni) più piccoli riguardano soprattutto la visione di film/serie TV e l'utilizzo di videogiochi, mentre quelle svolte dai ragazzi (11-18 anni) sono principalmente ascoltare musica e la messaggistica. I genitori tendono ad attribuire una quantità di tempo inferiore spesa sui media digitali dai loro figli di quella, in realtà, riportata da quest'ultimi. Sono emerse, infine, differenze significative legate al genere maschile, all'età preadolescenziale, al basso livello socio-economico, alla bassa scolarità materna e alla condizione di separati/divorziati, in direzione di una maggiore quantità di tempo trascorso su diversi me- 
dia digitali da parte dei partecipanti appartenenti a queste categorie.

Conclusioni - I risultati di questo studio contribuiscono ad ampliare le nostre conoscenze sull'utilizzo dei media digitali nei bambini e negli adolescenti, e possono fornire utili implicazioni di tipo clinico sulle modalità di intervento.

\section{INTRODUZIONE}

I bambini e gli adolescenti di oggi, i cosiddetti millennial o nativi digitali, trascorrono le loro vite immersi nel mondo dei media digitali. Il progressivo cambiamento che i media elettronici hanno apportato nella vita quotidiana ha subito un impulso dal 2007 con l'introduzione delle tecnologie touch, determinando una maggiore diffusione anche tra le fasce di età più piccole. Dati recenti di letteratura affermano che in Europa l'accesso domestico a internet è cresciuto dal 55\% nel 2007 all' $85 \%$ nel 2015 , e l'accesso a internet attraverso un dispositivo mobile è aumentato dal 36\% nel 2012 al 59\% nel $2016^{1}$. In aggiunta, secondo dati italiani, l'accesso a internet da parte di bambini e adolescenti avviene soprattutto da casa (88\%), con un progressivo incremento $(+13 \%)$ dell'uso quotidiano anche da parte dei più piccoli (9-10 anni), e un aumento degli accessi in mobilità (dal 30\% al 44\%) negli ultimi cinque anni ${ }^{2}$.

Una recente review australiana ${ }^{3}$ riporta come il 52,3\% di ragazzi tra i 5 e i 18 anni superi il tempo medio ( 2 ore) trascorso davanti a uno schermo (screen time) consigliato dalle principali linee guida internazionali ${ }^{4,5}$. Nello specifico, secondo ricerche internazionali, $\mathrm{i}$ tempi medi variano dalle 2,6 ore al giorno ${ }^{2,6}$ alle 3,6 ore $^{3,7}$, e picchi di utilizzo superiori alle 4 ore quotidiane nel $20 \%$ dei soggetti intervistati ${ }^{8}$. Infine, anche per la fascia di età compresa tra gli 0 e gli 8 anni, il tempo medio speso su un mobile device è triplicato da 5 minuti nel 2011 a 48 minuti nel $2017^{9}$. Il tempo trascorso davanti a uno schermo non rappresenta solo una fra le principali preoccupazioni di genitori ed educatori, che vedono il rischio che le attività online si sostituiscano alle relazioni vis-à-vis o ad altre attività, ma anche per chi si occupa a vario titolo di Salute pubblica (es. American Academy of Pediatrics) e di tecnologie digitali (come l'Autorità di regolazione dei Servizi di comunicazione del Regno Unito Ofcom e l'Institute of Digital Media and Child Development), date le conseguenze negative associate a un utilizzo eccessivo dei dispositivi digitali $^{10}$

A nostra conoscenza, non vi sono studi italiani che hanno analizzato la quantità di tempo impiegato dai bambini e dagli adolescenti nell'utilizzo di diversi media digitali, distinguendoli tra loro, unitamente al loro impatto sul funzionamento adattivo dei giovani fruitori.

Il presente studio si propone di indagare in modo dettagliato un ampio spettro di attività svolte tramite media digitali attraverso il questionario Media Activity Form $(\mathrm{MAF})^{11}$, costruito dal professor Thomas Achenbach della Università del Vermont. Si tratta di un breve questionario compilato dai genitori (MAF - G) e, a partire dagli 11 anni, dai ragazzi stessi (MAF - R), volto a valutare sia la frequenza delle diverse attività svolte tramite differenti media elettronici (quali ad esempio, internet, videogiochi, WhatsApp, Play Station, Xbox, YouTube), distinguendo il loro utilizzo in un giorno settimanale da quello nei fine settimana, sia l'impatto su alcune aree del funzionamento dei bambini e degli adolescenti.

L'obiettivo specifico di questa ricerca è quello di descrivere e analizzare l'utilizzo di diversi media elettronici e il loro impatto sul funzionamento adattivo in un ampio campione di bambini e adolescenti italiani, tra i sei e i diciotto anni, e i fattori di rischio sociodemografici a esso associati, attraverso il questionario MAF compilato sia dai genitori sia, a partire dagli 11 anni, dai ragazzi.

\section{METODI}

\section{Campione e procedura}

Il campione iniziale era costituito da 1431 soggetti, di età compresa tra i 6 e i 21 anni, reclutati attraverso 10 scuole, di ogni ordine e grado, della provincia di Monza Brianza, selezionate prevalentemente in modo casuale. Nello specifico, sono stati selezionati casualmente $6 \mathrm{Co}-$ muni, rappresentativi delle aree urbane (Monza, Vimercate) e semi-urbane (Lissone, Seregno, Lesmo, Besana in Brianza) secondo i dati dell'ISTAT. In collaborazione con l'Ufficio Scolastico Provinciale, all'interno di ciascun Comune sono state selezionate le scuole (laddove è stato possibile) e, all'interno di ciascuna scuola, una sezione. Al fine di avere una buona rappresentatività dei vari indirizzi di scuola secondaria di secondo grado, sono stati selezionati due licei e tre istituti tecnici. A ogni alunno delle classi selezionate è stata consegnata una busta pseudoanonimizzata, contenente i moduli di consenso e di trattamento dati, una scheda di raccolta dei dati sociodemografici e il questionario MAF da compilare a casa; a distanza di circa 10 giorni dalla consegna un ricercatore si recava a scuola per ritirare le buste compilate. I ragazzi della scuola secondaria di primo e secondo grado, previo consenso scritto dei genitori, hanno compilato il medesimo questionario in forma digitale, durante l'orario scolastico. I ragazzi maggiorenni hanno potuto scegliere in modo autonomo se partecipare al progetto compilando il questionario in forma digitale, e se coinvolgere anche il genitore nella compilazione del questionario. I dati dello studio sono stati raccolti e gestiti usando il sistema di raccolta dei dati elettronico REDCap ${ }^{12,13}$, in uso presso il nostro IRCCS.

La ricerca è stata preventivamente autorizzata dal Comitato etico dell'IRCCS Medea e tutti i partecipanti hanno firmato un modulo di consenso per iscritto.

Il campione finale di partecipanti è composto da 901 genitori e 574 ragazzi, con una percentuale di adesione rispettivamente del 63 e 65 percento, provenienti da aree urbane $(\mathrm{n}=361)$ e semi-urbane $(\mathrm{n}=594)$. In Tabella I sono riportate le frequenze e le percentuali dei partecipanti suddivisi in base a: genere, età, grado scolastico, livello 


\begin{tabular}{|c|c|}
\hline \multicolumn{2}{|c|}{ DESCRIZIONE DEL CAMPIONE (FREQUENZE E PERCENTUALI) } \\
\hline & Partecipanti $(n=955)$ \\
\hline $\begin{array}{l}\text { Genere } \\
M \\
\mathrm{~F}\end{array}$ & $\begin{array}{l}430(45 \%) \\
525(55 \%)\end{array}$ \\
\hline $\begin{array}{l}\text { Età } \\
6-11 \text { anni } \\
>12 \text { anni }\end{array}$ & $\begin{array}{l}424(44,4 \%) \\
531(55,6 \%)\end{array}$ \\
\hline $\begin{array}{l}\text { Grado scolastico } \\
\text { Scuola primaria di primo grado } \\
\text { Scuola secondaria di primo grado } \\
\text { Scuola secondaria di secondo grado }\end{array}$ & $\begin{array}{l}380(39,8 \%) \\
201(21,0 \%) \\
374(39,2 \%)\end{array}$ \\
\hline $\begin{array}{l}\text { Anni di studio della madre } \\
<\text { di } 10 \text { anni } \\
>\text { di } 10 \text { anni } \\
\text { Dati mancanti }\end{array}$ & $\begin{array}{c}227(23,8 \%) \\
715(74,9 \%) \\
13(1,4 \%)\end{array}$ \\
\hline $\begin{array}{l}\text { Anni di studio del padre } \\
<\text { di } 10 \text { anni } \\
>\text { di } 10 \text { anni } \\
\text { Dati mancanti }\end{array}$ & $\begin{array}{c}298(31,2 \%) \\
625(65,4 \%) \\
32(3,4 \%)\end{array}$ \\
\hline $\begin{array}{l}\text { Livello socioeconomico } \\
\text { Nessuna professione (disoccupati, pensionati, casalinghe) } \\
\text { Basso } \\
\text { Medio } \\
\text { Alto } \\
\text { Dati mancanti }\end{array}$ & $\begin{array}{c}3(0,3 \%) \\
153(16,0 \%) \\
436(45,7 \%) \\
331(34,7 \%) \\
32(3,4 \%)\end{array}$ \\
\hline $\begin{array}{l}\text { Stato civile dei genitori } \\
\text { Coniugati - conviventi } \\
\text { Separati - divorziati } \\
\text { Single - vedovi } \\
\text { Dati mancanti }\end{array}$ & $\begin{array}{c}806(84,4 \%) \\
113(11,8 \%) \\
26(2,7 \%) \\
10(1,0 \%)\end{array}$ \\
\hline $\begin{array}{l}\text { Nazionalità del genitore } \\
\text { Italiana } \\
\text { Non italiana } \\
\text { Dati mancanti }\end{array}$ & $\begin{array}{c}807(84,5 \%) \\
136(14,2 \%) \\
12(1,3 \%)\end{array}$ \\
\hline
\end{tabular}

\section{Tabella I}

di istruzione dei genitori, stato civile dei genitori, nazionalità dei genitori e livello socioeconomico (SES) - valutato attraverso la classificazione di Hollingshead ${ }^{14}$ che si basa sul tipo di professione svolta dai genitori.

\section{Strumenti}

Media Activity Form ${ }^{11}$. È stata utilizzata la versione italiana del MAF, curata da alcuni degli Autori (PC e AF) del presente articolo, autorizzata dal professor Achenbach attraverso una back-translation. Il questionario si compone di due parti: la prima valuta dettagliatamente quanto tempo (ore e minuti) il bambino o l'adolescente trascorre in diverse attività online (es. messaggistica, videogiochi, social network) durante un tipico giorno della settimana, il sabato e la domenica; la seconda analizza l'impatto dell'uso dei media digitali su alcune aree del funzionamento del ragazzo - come quella delle relazioni familiari (es. "Mio figlio farebbe più cose con i familiari se passasse meno tempo sui media"), della scuola (es. "Il lavoro scolastico di mio figlio sarebbe migliore se trascorresse meno tempo sui media"), del tempo libero (es. "Se mio figlio trascorresse meno tempo sui media, occuperebbe più tempo per gli sport, gli hobby o altre attività"), delle abitudini legate al sonno (es. "Mio figlio dormirebbe di più se passasse meno tempo sui media") - attraverso tredici item valutati su una scala Likert a 3 livelli $(0=$ non vero; 1 = in parte, qualche volta vero; 2 = molto vero, per lo più vero).

Esistono due versioni del questionario, una rivolta ai genitori di bambini e adolescenti di età compresa tra i $6 \mathrm{e}$ i 18 anni, l'altra rivolta ai ragazzi a partire dagli 11 fino ai 18 anni. L'unica differenza tra le due versioni del questionario è la possibilità di risposta "Non so" da parte del genitore, prevista per ogni item riguardante l'utilizzo dei media digitali. Nel nostro campione, la versione per genitori del questionario (MAF-G) è stata compilata nel $73,6 \%$ dei casi dalla madre, nel $16,1 \%$ dal padre, nel $9,7 \%$ da entrambi e solo da una percentuale inferiore al' $1 \%$ da altri componenti familiari.

In relazione alla prima parte del questionario, sono stati calcolati tre indicatori di tempo, espressi in minuti:

1. $\mathrm{MinWK}=$ tempo speso in un tipico giorno della settimana;

2. $\mathrm{MinWE}=$ tempo medio tra sabato e domenica trascorso in ogni attività;

3. MinTOT $=$ somma di tutti i minuti trascorsi per ogni attività.

Inoltre, le 13 attività indagate sono state raggruppate in 5 categorie:

1. Comunicazione (messaggistica, creazione contenuti, social network, parlare al telefono);

2. Uso di strumenti audiovisivi (ascoltare musica, guardare film/serie TV, guardare video su YouTube);

3. Videogiochi (videogiochi per adulti, giochi elettronici/ videogiochi);

4. Ricerca di informazioni per divertimento o altro (utilizzo dispositivi per scuola o altro, visitare siti web per divertimento);

5. Altro (giocare d'azzardo online, altro).

Per ciascuna categoria è stato calcolato il punteggio MinTOT.

Infine, in relazione alla seconda parte del questionario, è stato calcolato un punteggio globale (Imp) dato dalla somma di tutti i punteggi attribuiti ai 13 item per ottenere un valore complessivo dell'impatto.

\section{RISULTATI}

\section{Descrizione del tempo passato sui media} secondo il genitore e il ragazzo

La Tabella II riporta i tempi medi (espressi in minuti) trascorsi per ogni singola attività in un tipico giorno della settimana e nel fine settimana, sulla base delle risposte al MAF- G (6-11 e 11-18 anni) e al MAF-R. Per una maggiore leggibilità, $\mathrm{i}$ dati relativi a un tipico giorno della settimana vengono riportati anche in Figura 1.

Le attività principalmente svolte dai bambini tra i 6 e gli 11 anni sono la visione di film/serie TV, l'utilizzo di videogiochi, il guardare video su YouTube e ascoltare la musica, sia in un tipico giorno della settimana sia durante il weekend. In generale i tempi medi per queste attività non superano 1 ora, a eccezione della visione di film o serie TV nel fine settimana. Per quanto riguarda la fascia di età 11-18, secondo la percezione dei genitori, le attività prevalenti riguardano l'ascolto di musica, la messaggistica, l'uso di social network, la visione di film o serie TV; 
Rosi E, et al. Medico e Bambino 2021;24(2):e31-e38

https://www.medicoebambino.com/?id=RIC2102_10.html

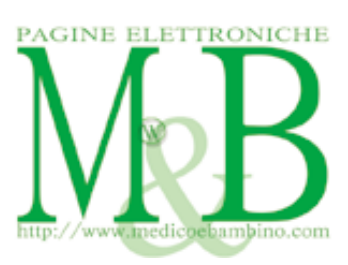

\begin{tabular}{|c|c|c|c|c|c|c|c|c|}
\hline \multicolumn{9}{|c|}{ STATISTICHE DESCRITTIVE (MEDIA E DEVIAZIONE STANDARD) DEGLI ITEM DEL MEDIA ACTIVITY FORM } \\
\hline & \multicolumn{6}{|c|}{ Versione del genitore } & \multirow{2}{*}{\multicolumn{2}{|c|}{$\begin{array}{c}\text { Versione del ragazzo } \\
11-18 \text { anni }\end{array}$}} \\
\hline & \multicolumn{3}{|c|}{ 6-11 anni } & \multicolumn{3}{|c|}{$11-18$ anni } & & \\
\hline & $\begin{array}{l}\text { In un giorno } \\
\text { della } \\
\text { settimana }\end{array}$ & Fine settimana & "Non so" & $\begin{array}{l}\text { In un giorno } \\
\text { della } \\
\text { settimana }\end{array}$ & Fine settimana & "Non so" & $\begin{array}{l}\text { In un giorno } \\
\text { della settimana }\end{array}$ & Fine settimana \\
\hline & M (DS) & M (DS) & $\%$ & M (DS) & M (DS) & $\%$ & M (DS) & M (DS) \\
\hline 1. Messaggistica & $5,8(37,3)$ & $4,4(22,9)$ & 2,4 & $74,1(105,1)$ & $83,8(120,1)$ & 7,6 & $121,7(177,3)$ & $116,5(138,8)$ \\
\hline 2. Creazione contenuti (es. blog) & $2,47(16,7)$ & $2,3(18,5)$ & 1,8 & $4,5(22,0)$ & $7,8(55,9)$ & 12,8 & $24,0(166,5)$ & $15,9(50,0)$ \\
\hline 3. Ascoltare musica & $14,68(32,4)$ & $20,6(32,9)$ & 1,8 & $83,4(97,7)$ & $89,8(104,9)$ & 4,5 & $128,0(198,3)$ & $113,6(131,0)$ \\
\hline 4. Videogiochi per adulti & $3,0(17,7)$ & $6,6(32,2)$ & 1,8 & $13,4(51,4)$ & $17,7(49,0)$ & 6,4 & $28,4(76,9)$ & $33,5(78,6)$ \\
\hline 5. Social network & $2,5(16,9)$ & $2,5(18,2)$ & 1,6 & $67,4(112,9)$ & $72,3(114,5)$ & 6,0 & $118,3(147,8)$ & $119,7(136,5)$ \\
\hline 6. Parlare al telefono & $4,3(13,1)$ & $4,2(11,6)$ & 2,6 & $53,9(103,6)$ & $50,3(93,4)$ & 6,0 & $85,3(155,7)$ & $76,5(125,8)$ \\
\hline 7. Giochi elettronici/videogiochi & $25,2(35,9)$ & $41,8(53,6)$ & 1,8 & $30,3(52,7)$ & $45,4(95,9)$ & 6,6 & $55,0(94,2)$ & $62,4(96,4)$ \\
\hline 8. Utilizzo device per scuola o altro & $13,4(25,9)$ & $14,1(28,5)$ & 4,5 & $59,7(72,5)$ & $41,7(57,9)$ & 8,6 & $72,3(117,2)$ & $52,8(69,0)$ \\
\hline 9. Siti web per divertimento & $12,2(26,7)$ & $14,4(31,6)$ & 3,4 & $34,5(40,8)$ & $33,2(53,8)$ & 11,7 & $45,5(74,5)$ & $41,6(54,7)$ \\
\hline 10. Guardare film/serie TV & $52,2(45,2)$ & $78,3(63,3)$ & 1,3 & $61,4(62,3)$ & $81,0(63,7)$ & 5,3 & $90,2(109,0)$ & $110,5(104,9)$ \\
\hline 11. Guardare video su YouTube & $27,7(37,4)$ & $37,8(49,3)$ & 2,1 & $47,4(57,7)$ & $56,8(99,2)$ & 10,7 & $76,6(131,0)$ & $73,6(99,0)$ \\
\hline 12. Giocare d'azzardo online & $0(0)$ & $0(0)$ & 1,8 & $0(0)$ & $0(0)$ & 5,3 & $2,43(19,8)$ & $2,0(16,8)$ \\
\hline 13. Altro & $1,0(6,4)$ & $1,2(9,5)$ & 3,7 & $0,8(8,2)$ & $0,9(9,3)$ & 13,6 & $31,8(87,9)$ & $83,3(255,1)$ \\
\hline \multirow{2}{*}{\multicolumn{3}{|c|}{ 14. Impatto sul funzionamento }} & \multicolumn{2}{|c|}{$\mathrm{M}(\mathrm{DS})$} & & & \multicolumn{2}{|c|}{$M(D S)$} \\
\hline & & & \multicolumn{2}{|c|}{$7,1(4,9)$} & & & \multicolumn{2}{|c|}{$8,2(4,3)$} \\
\hline
\end{tabular}

Tabella II

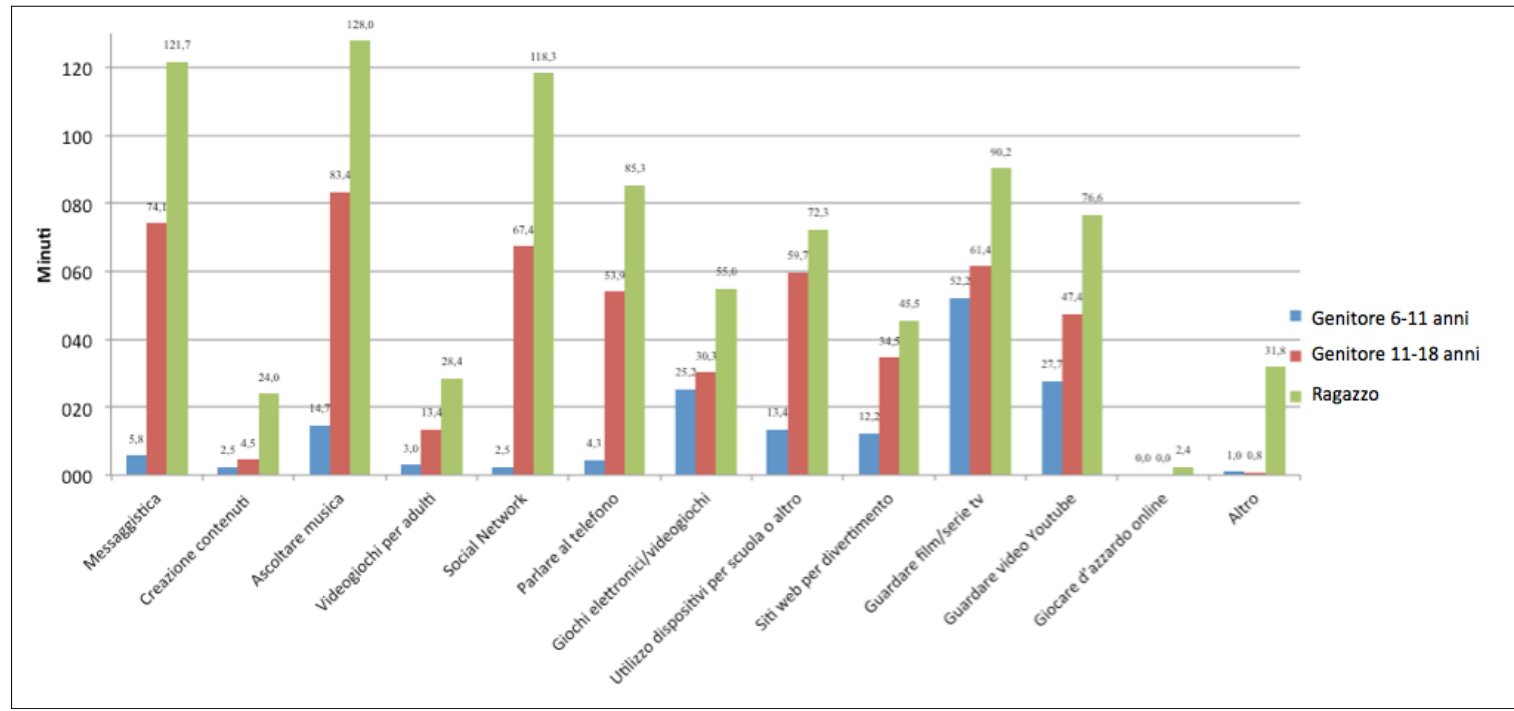

Figura 1. Media dei minuti trascorsi sui media digitali in un tipico giorno della settimana secondo il resoconto dei genitori e dei ragazzi.

secondo i ragazzi, ascoltare musica, mandare messaggi, i social network e la visione di film e serie TV.

Come riportato in Tabella II, il tempo medio riferito dai ragazzi per ciascuna di queste attività è molto maggiore rispetto a quanto riferito dai genitori, sia in un tipico giorno della settimana sia durante il weekend. In aggiunta, si osservano percentuali non trascurabili di genitori non in grado di quantificare il tempo che il proprio figlio/a trascorre nelle diverse attività, in particolare nella fascia di età 11-18 anni.

La percezione dei genitori rispetto all'impatto dell'uso dei media digitali sul funzionamento adattivo non si discosta in modo statisticamente significativo dalla autovalutazione del ragazzo $[\mathrm{t}(421)=1,003, \mathrm{p}=0,316]$. 


\section{Effetti delle variabili sociodemografiche sull'utilizzo dei media elettronici}

L'effetto delle variabili sociodemografiche (genere, età, livello socioeconomico, area geografica di provenienza, stato civile dei genitori, anni di scolarità dei genitori e Paese di provenienza dei genitori) su quelle dipendenti legate all'utilizzo dei media (i diversi item del questionario che indagano il tempo speso sui media e l'impatto) è stato analizzato attraverso l'analisi multivariata della varianza (MANOVA), per entrambi i questionari (MAF-G e MAF-R). La MANOVA è una tecnica di analisi dei dati che consente di verificare ipotesi relative a differenze tra le medie di due o più popolazioni, attraverso il test $\mathrm{F}$ (dato dal rapporto fra la varianza delle medie delle popolazioni a confronto).

È emerso un effetto significativo del genere sull'uso dei giochi elettronici, sia per quanto riguarda il questionario compilato dai genitori $[\mathrm{F}(1,461)=113,384 ; \mathrm{p}<0,001]$ sia per quello compilato dai ragazzi $[\mathrm{F}(1,209)=33,086$; $\mathrm{p}<0,001]$, nella direzione di un maggiore utilizzo dei maschi $(\mathrm{M}=568,6 ; \mathrm{DS}=610,9 ; \mathrm{M}=224,0 ; \mathrm{DS}=256,7)$ rispetto alle femmine $(\mathrm{M}=178,3 ; \mathrm{DS}=350,1 ; \mathrm{M}=$ $43,45 ; \mathrm{DS}=76,5)$. Anche per quanto riguarda l'impatto sul funzionamento adattivo, nel questionario compilato dai genitori, è risultato un effetto significativo del genere nella medesima direzione $[\mathrm{F}(1,420)=6,967 ; \mathrm{p}<0,01]$.

Per quanto concerne l'età, secondo la percezione dei genitori, sono i ragazzi della scuola secondaria di secondo grado che trascorrono più tempo nella comunicazione $[\mathrm{F}(2,460)=66,869 ; \mathrm{p}<0,001]$, nel ricercare informazioni per divertimento o altro $[\mathrm{F}(2,460)=60,926 ; \mathrm{p}<$ $0,001]$, e nell'uso di strumenti audiovisivi $[\mathrm{F}(2,460)=$ $33,969 ; \mathrm{p}<0,001]$, mentre i ragazzi della scuola secondaria di primo grado trascorrono più tempo sui videogiochi, sia rispetto ai ragazzi più grandi sia rispetto ai più piccoli $[\mathrm{F}(2,460)=4,205 ; \mathrm{p}<0,05]$, e subiscono maggiori conseguenze legate all'utilizzo delle attività digitali sul loro funzionamento rispetto ai bambini della scuola primaria $[F(1,420)=6,940 ; p<0,01]$. Secondo il resoconto del giovane, i ragazzi della scuola secondaria di secondo grado trascorrono più tempo nella comunicazione $[\mathrm{F}(1,209)$ $=8,367 ; \mathrm{p}<0,01]$ e nella ricerca di informazioni $[\mathrm{F}$ $(1,209)=5,538 ; \mathrm{p}<0,05]$.

Per quanto concerne l'effetto del livello socioeconomico sui punteggi al questionario compilato dai ragazzi, emerge come i partecipanti con un basso SES trascorrano in media più tempo nella attività comunicative $[\mathrm{F}(2,200)$ $=4,020 ; \mathrm{p}<0,05]$, nell'uso di strumenti audiovisivi $[\mathrm{F}(2$, $200)=4,933 ; \mathrm{p}<0,01]$ e nella ricerca di informazioni per divertimento o altro $[\mathrm{F}(2,200)=3,443 ; \mathrm{p}<0,05]$, rispetto a coloro che hanno un livello socio-economico elevato. Tale tendenza si conferma anche nella versione compilata dal genitore, in relazione all'attività di comunicazione $[\mathrm{F}(2,449)=5,647 ; \mathrm{p}<0,01]$, mentre i partecipanti con un livello sia basso sia medio trascorrono più tempo sui giochi elettronici $[\mathrm{F}(2,449)=3,459 ; \mathrm{p}<0,05]$ e nella ricerca di informazioni $[\mathrm{F}(2,449)=3,442 ; \mathrm{p}<0,05]$.
Sono emersi, inoltre, effetti significativi legati al livello di educazione materna e paterna sull'utilizzo di diversi media digitali. In particolare, un basso livello di istruzione (inferiore a 10 anni) della madre ha un effetto significativo, sia nella percezione del genitore sia in quella del giovane, sull'aspetto della comunicazione $[\mathrm{F}(1,459)=$ 8,495; $\mathrm{p}<0,01 ; \mathrm{F}(1,207)=19,140 ; \mathrm{p}<0,001]$, sull'uso di strumenti audiovisivi $[\mathrm{F}(1,459)=9,801 ; \mathrm{p}<0,01 ; \mathrm{F}(1$, $207)=11,501 ; \mathrm{p}<0,001]$, sulla ricerca di informazioni $[\mathrm{F}(1,459)=10,131 ; \mathrm{p}<0,01 ; \mathrm{F}(1,207)=4,193 ; \mathrm{p}<$ $0,05]$ e, solo nella versione compilata dal genitore, sull'utilizzo di altri dispositivi o di altre attività online $[\mathrm{F}(1,459)=13,628 ; \mathrm{p}<0,001]$, nella direzione di un tempo maggiore trascorso in queste attività. Il basso livello di istruzione materno è inoltre associato a un peggiore funzionamento adattivo del ragazzo, nella versione MAF$\mathrm{G}[\mathrm{F}(1,417)=6,612 ; \mathrm{p}<0,01]$. Invece, un basso livello di istruzione del padre si associa, solo nella versione del questionario compilata dal genitore, a una maggiore quantità di tempo trascorsa nelle attività di comunicazione $[\mathrm{F}(1,452)=19,858 ; \mathrm{p}<0,001]$, sull'uso di strumenti audiovisivi $[\mathrm{F}(1,452)=15,462 ; \mathrm{p}<0,001]$, nella ricerca di informazioni $[\mathrm{F}(1,452)=5,972 ; \mathrm{p}<0,05]$ e nell'utilizzo di altri dispositivi o tipologia di attività online $[\mathrm{F}(1,452)=$ 4,817; $\mathrm{p}<0,05]$.

Infine, secondo la percezione del ragazzo, non emergono effetti significativi legati allo stato civile dei genitori sull'utilizzo delle diverse attività digitali, mentre, secondo quella del genitore, emerge un effetto significativo sull'attività di comunicazione $[\mathrm{F}(2,458)=3,229 ; \mathrm{p}<$ $0,05]$ e sull'utilizzo dei giochi elettronici $[\mathrm{F}(2,458)=$ $6,530 ; p<0,01]$. Nello specifico, secondo la percezione del genitore, i figli di separati o divorziati trascorrono più tempo svolgendo queste attività rispetto ai loro coetanei che hanno genitori coniugati o conviventi e hanno in genere maggiori conseguenze sul loro funzionamento $[\mathrm{F}$ $(2,418)=4,016 ; \mathrm{p}<0,05]$.

\section{DISCUSSIONE}

I risultati del nostro studio offrono un quadro dettagliato sulla quantità di tempo che $\mathrm{i}$ bambini e gli adolescenti trascorrono sui vari media digitali e il conseguente impatto sul loro funzionamento, in base alla somministrazione di un nuovo questionario, il MAF, in un ampio campione di genitori e ragazzi residenti nella provincia di Monza Brianza.

Le attività digitali che $\mathrm{i}$ pre/adolescenti privilegiano sono principalmente ascoltare musica, mandare messaggi, guardare film e serie TV, e utilizzare i social network, in linea con quanto emerge dai risultati di altri studi naziona$\mathrm{li}^{2,15}$ e internazionali ${ }^{7}$. Va sottolineato, inoltre, che il tempo riferito dai ragazzi per molte delle attività indagate si aggira intorno alle 2 ore, per cui è possibile dedurre che lo screen time totale di una giornata sia largamente superiore a quello indicato dalle principali linee guida internaziona$1 i^{4,5}$. Tale risultato suggerisce l'importanza di svolgere ulteriori indagini per comprendere se e quali siano le conse- 
guenze per il benessere dei ragazzi di un utilizzo così massiccio dei media digitali, anche attraverso il coinvolgimento diretto dei pediatri di famiglia ${ }^{16-18}$. Mancano, difatti, a oggi, delle linee guida italiane sull'utilizzo dei media digitali nella fascia di età preadolescenziale e adolescenziale, mentre sono presenti in riferimento ai bambini più piccoli ${ }^{15,19}$.

Per quanto riguarda la fascia di età compresa tra i 6 e gli 11 anni, i genitori riportano che i figli, già a partire dai 6 anni, utilizzano i media digitali per svolgere quasi tutte le attività indagate, seppure con tempi medi inferiori rispetto ai ragazzi più grandi, e con alcune preferenze che li caratterizzano: guardare film e serie TV, guardare video su YouTube, giocare con giochi elettronici, ascoltare musica. Tra i bambini più piccoli le attività che riguardano la comunicazione sono comunque presenti, anche se in misura più ridotta. Nonostante i tempi medi di utilizzo dei media digitali siano inferiori alle 2 ore suggerite dalle linee guida nazionali $^{19} \mathrm{e}$ internazionali ${ }^{4,5}$, il dato che colpisce riguarda il fatto che l'interazione con il mondo digitale nei bambini più piccoli si caratterizza per un'ampia gamma di attività, non solo di fruizione di contenuti, ma anche di comunicazione e interazione sociale. Tale dato si pone in linea con i risultati di recenti studi italiani ${ }^{20,21}$ che rilevano un precoce utilizzo dei media digitali nei bambini fin dall'età prescolare, mettendone in luce le problematicità.

Questa indagine ci ha, inoltre, consentito di confrontare le percezioni che ragazzi e genitori hanno in merito a tali attività. Nonostante ci sia generalmente accordo sulle tipologie di attività svolte, è interessante notare come la quantificazione del tempo impiegato in ciascuna attività sia molto diversa tra ragazzi e genitori, nettamente sottostimata da questi ultimi. Non sono, invece, emerse differenze statisticamente significative tra genitori e figli rispetto alle conseguenze dell'uso dei media elettronici. Pertanto, è possibile rilevare come, indipendentemente dalla quantità di tempo speso nel loro utilizzo (differente nella percezione dei genitori rispetto a quanto riferito dai figli), adulti e ragazzi attribuiscano lo stesso impatto dell'uso dei media digitali sul funzionamento in ambito scolastico, sociale e familiare. Occorre, tuttavia, sottolineare che non sono ancora disponibili dei valori normativi di riferimento del questionario MAF che consentano di definire con chiarezza quando l'impatto dell'utilizzo dei media digitali sul funzionamento del bambino $\mathrm{e}$ dell'adolescente diventa clinicamente significativo.

È stato, inoltre, analizzato l'effetto dei fattori sociodemografici sull'utilizzo dei media digitali. In linea con i dati presenti in letteratura ${ }^{2,3,22,23}$, i maschi trascorrono più tempo delle femmine sui videogiochi, mentre, contrariamente alle nostre aspettative, quest'ultime non trascorrono più tempo dei loro coetanei maschi nelle attività di comunicazione (uso di social network, messaggistica e parlare al telefono) come invece viene riportato da altri studi $^{2,23}$ che considerano separatamente le diverse attività di comunicazione, individuando una predilezione per l'uso dei social media da parte del genere femminile. Abbiamo inoltre osservato come all'aumentare dell'età vi sia un incremento nell'utilizzo di diversi media digitali ${ }^{2,24}$, a eccezione dell'uso dei videogiochi che risulta, secondo la percezione dei genitori, più massiccio tra i ragazzi della scuola secondaria di primo grado rispetto non solo ai bambini della scuola primaria ma anche ai ragazzi più grandi. Questo dato, potrebbe suggerire un rischio più alto legato a un uso eccessivo dei videogiochi in preadole$\operatorname{scenza}^{25}$. In aggiunta, i genitori, a differenza dei figli, riportano come siano i maschi e i ragazzi delle medie a subire più conseguenze sul loro funzionamento associate all'utilizzo dei media digitali. Tale dato è in linea con la letteratura, che considera il genere maschile un fattore di rischio per lo sviluppo di un uso patologico di internet ${ }^{26-28}$ e l'età preadolescenziale come una fascia più vulnerabile per lo sviluppo della dipendenza dai videogiochi, ${ }^{6,25}$. Inoltre, va sottolineato come questi risultati, emersi esclusivamente dal resoconto del genitore, potrebbero riflettere una maggiore preoccupazione da parte dei genitori per $\mathrm{i}$ figli maschi e per i figli preadolescenti, fascia di età in cui i ragazzi iniziano in generale a usare i media digitali con maggiore frequenza e autonomia.

Per quanto riguarda i risultati relativi al SES, i nostri dati indicano che un basso SES è legato a un maggior utilizzo dei media digitali da parte dei ragazzi. I nostri dati sono in linea con quelli di uno studio che ha trovato un'associazione tra basso SES e vita sedentaria, variabile che include anche il tempo speso su attività online ${ }^{29}$. Altri studi, al contrario, hanno riscontrato come siano i ragazzi con un alto livello socio economico a trascorrere più tempo sui media digitali verosimilmente perché hanno maggiori disponibilità di mobile device nel contesto familia$\mathrm{re}^{24,26}$ o non hanno rilevato alcun effetto del $\mathrm{SES}^{30}$. È possibile che i risultati così contrastanti siano da attribuire a differenze metodologiche (legate ad esempio all'ampiezza del campione, all'età dei partecipanti, agli strumenti di misurazione del SES o dell'uso dei media) tra gli studi.

Inoltre, un livello di educazione genitoriale inferiore ai dieci anni di scolarità, specialmente materna, è legato a un maggior utilizzo dei media digitali da parte dei ragazzi e, nella percezione del genitore, ha un'influenza sull'attribuzione dell'impatto sul funzionamento del ragazzo. Un recente studio $^{31}$ sulle problematiche connesse all'uso di internet mette in luce come un alto livello di educazione materna sia un fattore protettivo rispetto a un utilizzo patologico di internet. Seppure con alcune differenze di metodo, anche i nostri dati evidenziano un ruolo rilevante dell'educazione materna sia sui tempi di utilizzo sia sull'impatto; il ruolo del livello di educazione paterna è più difficilmente interpretabile, in quanto da un lato i nostri dati sono meno consistenti (troviamo infatti meno effetti significativi e solo nel MAF-G), e dall'altro è un fattore meno studiato in letteratura.

Infine, i risultati del nostro studio indicano come i genitori separati o divorziati riferiscano maggiori tempi di utilizzo dei media da parte dei loro figli rispetto a quanto riferito dai genitori coniugati o conviventi, in continuità con altre ricerche che hanno evidenziato un'associazione tra un utilizzo patologico di internet e relazioni genitoriali 
negative o divorzio dei genitori ${ }^{22,32}$. Nonostante ci siano pochi elementi per poter dare un'interpretazione accurata, questo dato potrebbe riflettere sia un effettivo maggiore utilizzo dei media digitali da parte dei figli di divorziati o separati, tendenza messa in luce da precedenti studi ${ }^{22,32}$, sia una percezione differente dei genitori divorziati o separati rispetto al tempo speso in attività online da parte dei loro figli, in quanto tale differenza non emerge dalle risposte dei ragazzi.

Lo studio presenta alcuni limiti, che debbono essere tenuti in considerazione. Primo, non sono ancora disponibili dati sui valori normativi di riferimento dello strumento, oggetto a breve di un'altra pubblicazione. Secondo, i partecipanti sono stati selezionati solo in scuole pubbliche, ma un numero non trascurabile di bambini e adolescenti italiani frequenta strutture private. Infine, non abbiamo informazioni sui soggetti che non hanno dato il loro consenso alla partecipazione allo studio, per cui non possiamo escludere che vi siano delle differenze nell'utilizzo dei media digitali rispetto ai partecipanti.

Nonostante i limiti sopra riportati, questa ricerca contribuisce a ampliare le nostre conoscenze in merito all'utilizzo di diversi tipologie di media digitali in un ampio campione di bambini e adolescenti, tenendo conto di fattori - come il genere, l'età, il livello socioeconomico e lo stato civile dei genitori - che possono condizionare la quantità di tempo speso su diversi tipi di attività, attraverso uno strumento che consente di confrontare il punto di vista del genitore e del ragazzo. Il questionario, una volta completata la taratura, potrà essere proposto come un valido e sostenibile strumento di raccolta di informazioni durante le visite routinarie dei bilanci di salute, richiamando così l'attenzione di genitori e figli verso un'educazione a un uso appropriato dei media digitali fin dai primi anni di vita.

\section{BIBLIOGRAFIA}

[1] Eurostat. Digital economy and society statistics households and individuals. 2017.

[2] Mascheroni G, Ólafsson K. Accesso, usi, rischi e opportunità di internet per i ragazzi italiani. I risultati di EU Kids Online 2017. EU Kids Online e OssCom 2018.

[3] Thomas G, Bennie JA, De Cocker K, Castro O, Biddle SJH. A descriptive epidemiology of screenbased devices by children and adolescents: a scoping review of 130 surveillance studies since 2000. Child Ind Res 2019;12:1-16. DOI: 10.1007/s12187 -019-09663-1.

[4] Tremblay MS, Carson V, Chaput JP, et al. Canadian 24-hour movement guidelines for children and youth: an integration of physical activity, sedentary behaviour, and sleep. Appl Physiol Nutr Metab 2016;41:S311-27. DOI: 10.1139/apnm2016-0151.
[5] Australian Government Department of Health. Make your move - Sit less - Be active for life! Canberra: Commonwealth of Australia, 2014.

[6] Pontes HM. Investigating the differential effects of social networking site addiction and Internet gaming disorder on psychological health. J Behav Addict 2017;6:601-10. DOI: 10.1556/2006.6.2017.075.

[7] Hussain Z, Griffiths MD, Sheffield D. An investigation into problematic smartphone use: the role of narcissism, anxiety, and personality factors. J Behav Addict 2017;6:378-86. DOI: 10.1556/2006.6. 2017.052.

[8] Vismara MFM, Toaff J, Pulvirenti G, et al. Internet use and access, behavior, cyberbullying, and grooming: results of an investigative whole city survey of adolescents. Interact J Med Res 2017;6: 1-14. DOI: 10.2196/ijmr.6231.

[9] Rideout V. The common sense census: media use by kids age zero to eight. San Francisco, CA: Common Sense Media, 2017, pagg. 263-83.

[10] Carson V, Hunter S, Kuzik N, et al. Systematic review of sedentary behaviour and health indicators in school-aged children and youth: an update. Appl Physiol Nutr Metab 2016;41:S240-S265. DOI: 10.1139/apnm-2015-0630.

[11] Achenbach T. Media Activity Form (MAF). 2018 [Unpublished].

[12] Harris PA, Taylor R, Thielke R, Payne J, Gonzalez N, Conde JG. Research electronic data capture (REDCap) - a metadata-driven methodology and workflow process for providing translational research informatics support. J Biomed Inform 2009; 42:377-81. DOI: 10.1016/j.jbi.2008.08.010.

[13] Harris PA, Taylor R, Minor BL, et al.; REDCap Consortium. The REDCap consortium: Building an international community of software partners. J Biomed Inform 2019;95:103208. DOI: 10.1016/j. jbi.2019.103208.

[14] Hollingshead AB. Four factor index of social status. Unpublished manuscript. New Haven, CT: Yale University, 1975.

[15] Qualizza G. Giovani e nuovi media: pratiche di consumo digitale e dinamiche relazionali. Medico e Bambino 2012;31(10):639-46.

[16] Tamburlini G. Tecnologie digitali e bambini Indicazioni per un utilizzo consapevole. Medico e Bambino 2015;34(1):31-8.

[17] Tamburlini G. Bambini e tecnologie digitali: modificazioni del costume, opportunità e rischi. Medico e Bambino 2012;31(10):619-20.

[18] Tamburlini G. La follia digitale precoce. Medico e Bambino 2017;36(3):143.

[19] Bozzola E, Spina G, Ruggiero M, et al. Bambini in età prescolare e media device: le raccomandazioni della Società Italiana di Pediatria. 2018. 
[20] Bozzola E, Spina G, Ruggiero M, et al. Media devices in pre-school children: the recommendations of the Italian pediatric society. Ital J Pediatr 2018; 44:69-75. DOI: 10.1186/s13052-018-0508-7.

[21] Balbinot V, Toffol G, Tamburlini G. Tecnologie digitali e bambini: un'indagine sul loro utilizzo nei primi anni di vita. Medico e Bambino 2016;35(10): 631-6.

[22] Anderson EL, Steen E, Stavropoulos V. Internet use and problematic internet use: a systematic review of longitudinal research trends in adolescence and emergent adulthood. Int J Adolesc Youth 2017;22:430-54. DOI: 10.1080/02673843.2016.1227716.

[23] Scimeca G, Bruno A, Cava L, Pandolfo G, Muscatello MRA, Zoccali R. The relationship between alexithymia, anxiety, depression, and internet addiction severity in a sample of Italian high school students. The Scientific World Journal 2014; 504376: 1-8. DOI: 10.1155/2014/504376.

[24] Smahel D, Helsper E, Green L, Kalmus V, Blinka L, Ólafsson K. Excessive internet use among European children. London: EU Kids Online, 2012.

[25] Amendola S, Spensieri V, Cerutti R. L'uso dei videogiochi. Medico e bambino 2019;38(3):177-82.

[26] Kuss JD, Griffiths DM, Karila L, Billieux J. Internet addiction: a systematic review of epidemiological research for the last decade. Curr Pharm Des 2014;20:4026-52. DOI: $10.2174 / 13816128113199990617$.
[27] Kuss DJ, Lopez-Fernandez O. Internet addiction and problematic internet use: a systematic review of clinical research. World J Psychiatry 2016;6: 143-76. DOI: 10.5498/wjp.v6.i1.143.

[28] Carli V, Durkee T, Wasserman D, et al. The association between pathological internet use and comorbid psychopathology: a systematic review. Psychopathology 2013;46:1-13. DOI: 10.1159/ 000337971.

[29] Tsitsika AK, Andrie EK, Psaltopoulou T, et al. Association between problematic internet use, sociodemographic variables and obesity among European adolescents. Eur J Public Health 2016;26: 61722. DOI: 10.1093/eurpub/ckw028.

[30] Mascheroni G, Ólafsson K. The mobile internet: access, use, opportunities and divides among European children. New Media Soc 2016;18:1657-79. DOI: $10.1177 / 1461444814567986$.

[31] Lai FJ. Socioeconomic influence on adolescent problematic Internet use through school-related psychosocial factors and pattern of Internet use. Comput Human Behav 2017;68:121-36. DOI: 10. 1016/j.chb.2016.11.021.

[32] Willoughby T. A short-term longitudinal study of Internet and computer game use by adolescent boys and girls: prevalence, frequency of use, and psychosocial predictors. Dev Psychol 2008;41:95204. DOI: 10.1037/0012-1649.44.1.195. 